\title{
How to apply for a research grant: 10 tips and tricks
}

\author{
L. Harper, M. Castagnetti, K. Herbst, D. Bagli, M. Kaefer, G. Beckers, M. Fossum and N. Kalfa
}

Research requires money. Many research grants are available, yet competition is fierce. Here are some tips that can help make your application successful.

1) Read the funding body's instructions and know their interests. Your proposal might be rejected simply because of misalignment with the grantor's interests and mandate. When responding to a grant request, highlight how your idea meets their criteria throughout the application. It is important to understand what can or cannot be funded on a particular call. In case of doubt, contact the funding body for clarification.

2) Find a good research question, especially one that interests you. Read the published literature, highlight debated topics, and consider new tools and approaches for unanswered questions. Choose a topic that falls within your area of expertise as experience and prior publications give credibility. Explain why your research question is important and how it may lead to change clinical practice.

3) Keep it simple. Your study question should be focused. This limits the risk of bias and contestable designs. Complex questions often cannot be answered with a feasible study design, and compound questions can be confusing and dilute effects. Secondary objectives are important. They should use the same method and population and require minor additional analysis or extra work.

4) Become familiar with grant writing early. Participate in local courses on research and in work done by senior researchers. Apply for local grants first, and then graduate to national/international grants as each process teaches you something about refining your protocol.

This is the author's manuscript of the article published in final edited form as:

Harper, L., Castagnetti, M., Herbst, K., Bagli, D., Kaefer, M., Beckers, G., ... Kalfa, N. (2018). How to apply for a research grant: 10 tips and tricks. Journal of Pediatric Urology, 14(5), 453-454.

https://doi.org/10.1016/j.jpurol.2018.08.016 
5) Be realistic about financial budgets. Decide what you need the money for, what will be outside the standard care, and what resources you already have. Expect additional spending for unexpected problems such as indirect administrative costs. Asking for too little appears unprepared or naive.

6) Give yourself plenty of time. It takes a lot of time to prepare a protocol and to get input from all the experts.

7) Stay focused and avoid jargon. Explain why you are the right person with the right blend of skills and experience. Get your sums right (number of patients that can be included, etc...). Be clear and precise. Wordings should be understood also by non-specialists in your field. Do not exceed page limitations or maximum word count and do not miss any required attachments.

8) Talk over interdisciplinary proposals with your collaborators, involve them as soon as possible. Collaborative projects take longer. Multicenter studies require standardization of clinical practices.

9) Get help from your research department, colleagues, and even friends and family who can read your application and identify areas for improvement. In some countries, patient representatives are now appearing on grant's panels, and it can help to have support from patient self-help groups or organizations.

10) If you get rejected, try again. Rejection rates are very high (80\%). Reviewer comments often lead to substantial improvements, and applications can go on to be funded. Try out a set of ideas several times. Consider an alternative approach or angle. As Churchill said, "Success is the ability to go from failure to failure without losing enthusiasm." 